\title{
ENDOPARASITOS DE Myrmecophaga tridactyla Y Tamandua tetradactyla (PILOSA: VERMILINGUA) SILVESTRES EN CASANARE, COLOMBIA
}

\author{
ENDOPARASITES IN WILD Myrmecophaga tridactyla AND Tamandua \\ tetradactyla (PILOSA: VERMILINGUA) IN CASANARE, COLOMBIA
}

\author{
CESAR ROJANO,${ }^{1 *} \mathrm{MVZ}$, LAURA MIRANDA C,${ }^{1}$ Ecol, RENZO ÁVILA A,${ }^{1}$ Ecol.
}

${ }^{1}$ Fundación Cunaguaro. Proyecto de conservación del oso palmero. Carrera $22 \mathrm{~N}^{\circ} 8-28$. Yopal - Casanare.

\section{Key words:}

parasites, Vermilingua,

Orinoquia,

protozoa,

cestodes

\begin{abstract}
Endoparasites of 11 giant anteaters (Myrmecophaga tridactyla) and two southern tamanduas (Tamandua tetradactyla) present in livestock areas of the municipality of Pore, Casanare, Colombia, were determined. Blood and feces of giant anteaters anesthetized on field were collected while southern tamandua's feces samples were collected from individuals killed on roads of the area. Feces were analyzed by sedimentation and flotation techniques and hemoparasites were determined by thick smear technique. Seven M. tridactyla (63.6\%) and both T. tetradactyla (100\%) were positive for gastrointestinal parasites. The prevalence of each parasite in giant anteaters was: Coccidia sp (28\%), Blastocistis hominis (28\%), Entomoeba dispar(14.28\%), Giardia lamblia(14.28\%), Isosporasp. (14.28\%), Strongyloides sp. (14.28\%), Ascaris lumbricoides (14.28\%) and Chilomastix mesnili(14.28\%). As for southem tamanduas, the most prevalent parasite was Coccidia (100\%), while Trichostrongylus sp., and Taenia sp., had a prevalence of $50 \%$. The monoxenas parasitic forms were predominant on those with multiple hosts cycles, probably for the direct consumption of land and/or water contaminated with fecal material. This is the first report of parasites occurrence in wild Vermilingua in Colombia. Although the sample sizes are not significant, and there is not enough evidence to say that these parasitic infestations may be severely affecting the sampled individuals, this information may be useful for reintroduction programs and management of these species.
\end{abstract}

\section{Resumen}

Se determinaron los endoparásitos de 11 osos hormigueros gigantes (Mymecophaga tridactyla) y dos ososmeleros(Tamanduatetradactyla) presentesenzonasganaderas del municipiodePore, Casanare, Colombia. Serecolectaronmuestras desangreyheces dehomiguerosgigantesanestesiadosencampo, mientras que las muestras de heces de osos meleros fueron recolectadas de individuos atropellados en la zona. Se analizaron las heces mediante técnicas de sedimentación y flotación, y se determinaron hemoparásitos mediante la técnica de gota gruesa. Siete $M$. tridactyla $(63,6 \%)$ y ambos $T$. tetradactyla (100\%)seencontraron positivosaalgunaespeciedeparásitogastrointestinal. Laprevalenciadecadauno delosparásitosidentificadosen hormigueros gigantesfue: Coccidiasp(28\%), Blastocistishominis(28\%), Entomoebadispar(14,28\%), Giardialamblia(14,28\%), Isosporasp.(14,28\%), Strongyloidessp.(14,28\%), Ascaris/umbricoides(14,28\%)y Chilomastixmesnill(14,28\%). Encuantoalosososmeleros, la Coccidia fueel parásitomás prevalente(100\%), mientras que Trichostrongylussp., y Taeniasp., presentaron una prevalenciadel $50 \%$. Lasformasparásitasmonoxenasfueron predominantessobreaquellasquetienen ciclos de varios huéspedes, probablemente por el consumo directo de tierra y/o agua contaminada con material fecal. Se presenta el primer reporte de parásitos en Vermilingua silvestres en Colombia. Si bien los tamaños de muestra no son significativos, y no se cuenta con evidencia suficiente para afirmar que estasinfestaciones parasitarias puedanestarafectandodemanera severaalosindividuosmuestreados, esta información puede ser de utilidad para programas de reintroducción y manejo de estas especies. 


\section{Introducción}

Los osos hormigueros gigantes (Myrmecophaga tridactyla) y osos meleros (Tamandua tetradactyla) son mamíferos del suborden Vermilingua distribuidos en amplias zonas del Neotrópico (SUPERINA et al., 2010). El hormiguero gigante se encuentra categorizado a nivel nacional como vulnerable a la extinción debido a una reducción de aproximada del $30 \%$ de su población en los últimos años por causa de la pérdida de hábitat, atropellamientos y quemas (RODRÍGUEZ et al., 2006).

Por su parte el oso melero no está categorizado en el país dentro de las especies amenazadas de extinción; sin embargo, los ataques de perros, la pérdida y degradación del hábitat y los atropellamientos representan amenazas considerables en algunas áreas (SUPERINA et al., 2010). Otro aspecto a considerar es que no se conoce el efecto del contacto con el ganado y otra fauna domestica sobre la salud de las poblaciones silvestres, ni cómo afecta a los hormigueros el contacto con agroquímicos y pesticidas (RODRÍGUEZ et al., 2006).

Dentro de este contexto, las enfermedades infecciosas son una amenaza emergente que los conservacionistas están mal preparados para manejar. La amenaza es mayor para las poblaciones pequeñas, que pueden ser llevadas a la extinción por agentes patógenos virulentos, que son por lo general los patógenos generalistas que llegan a ser dispersados por otras especies, a menudo animales domésticos (WOODROFFE, 1999). Es por esto que se considera que el riesgo de morbilidad y mortalidad por causa de enfermedades infecciosas es un tema significativo para la conservación de la fauna silvestre en general.

Una prevalencia aumentada de determinadas patologías están más asociadas con la escala global de cambios antropogénicos, que incluyen el crecimiento de las poblaciones humanas, fragmentación y degradación, el aislamiento de las poblaciones de las especies y la proximidad a los seres humanos (y sus animales domésticos) a la vida silvestre (DEEM et al., 2001). Dentro de las enfermedades infecciosas se destacan las parasitosis, las cuales tienen una gran importancia clínica. En Vermilinguas, los parásitos intestinales más frecuentes son los protozoarios, destacándose la coccidiosis, giardiasis y amebiasis (MIRANDA y DE PAULA, 2013). Sin embargo, son pocos los estudios realizados en vida silvestre sobre la flora parasitaria de T. tetradactyla y M. tridactyla.

El objetivo de este estudio fue identificar los parásitos gastrointestinales y hemáticos del oso hormiguero gigante y el oso melero muestreados en zonas ganaderas del municipio de Pore, Casanare, Colombia.

\section{Materiales y métodos}

Este estudio se desarrolló en el municipio de Pore, Casanare, en fincas ganaderas de las veredas San Rafael $\left(5.363^{\circ} \mathrm{N} 71.474^{\circ} \mathrm{W}\right)$ y Cafifíes $\left(5.342^{\circ} \mathrm{N}\right.$, $71.443^{\circ} \mathrm{W}$ ), entre los meses de julio y noviembre de 2014. La región presenta una temperatura promedio de $27^{\circ} \mathrm{C}$ y humedad relativa promedio del $75 \%$. El ecosistema predominante es la sabana natural inundable, aunque también se encuentran grandes extensiones de arrozales y pasturas introducidas para el ganado. El tamaño de la muestra se estimó por conveniencia debido a las dificultades de captura de individuos silvestres de estas especies.

Para la captura de los 11 hormigueros gigantes adultos, sujetos de estudio, en estado silvestre, se utilizó el método de búsqueda activa del animal a pie o a caballo y posteriormente se inmovilizaron aplicando un dardo con un coctel anestésico consistente en Ketamina a dosis de 12 $\mathrm{mg} / \mathrm{kg}$ y Xilacina $0,5 \mathrm{mg} / \mathrm{kg}$. Luego de ser anestesiados, se tomaron muestras fecales por medio de hisopados rectales o colecta de muestras frescas que el animal defecara durante el procedimiento. Al mismo tiempo se recolectaron hasta $5 \mathrm{ml}$ de sangre de cada individuo a través de punción de la vena safena o braquial, con el uso de tubos al vacío (vacutainers $₫$ ). Cada muestra fue mantenida en tubos con EDTA para luego ser procesadas. De igual forma, se colectó material fecal de dos osos meleros que fueron encontrados muertos por atropellamiento en las carreteras del municipio de Pore. Los locales de colecta de muestras se encuentran en la Tabla 1.

Tabla 1. Coordenadas de captura de M. tridactyla y T. tetradactyla

\begin{tabular}{ccc}
\hline \multirow{2}{*}{ \#individuo } & \multicolumn{2}{c}{ Local de captura } \\
& Oeste & Norte \\
\hline MT1 & $71.771^{\circ}$ & $5.644^{\circ}$ \\
MT2 & $71.795^{\circ}$ & $5.609^{\circ}$ \\
MT3 & $71.740^{\circ}$ & $5.572^{\circ}$ \\
MT4 & $71.824^{\circ}$ & $5.603^{\circ}$ \\
MT5 & $71.827^{\circ}$ & $5.601^{\circ}$ \\
MT6 & $71.819^{\circ}$ & $5.603^{\circ}$ \\
MT7 & $71.820^{\circ}$ & $5.604^{\circ}$ \\
MT8 & $71.734^{\circ}$ & $5.574^{\circ}$ \\
MT9 & $71.745^{\circ}$ & $5.583^{\circ}$ \\
MT10 & $71.729^{\circ}$ & $5.572^{\circ}$ \\
MT11 & $71.799^{\circ}$ & $5.603^{\circ}$ \\
TT1 & $72.177^{\circ}$ & $5.550^{\circ}$ \\
TT2 & $71.963^{\circ}$ & $5.694^{\circ}$ \\
\hline
\end{tabular}

Las muestras fecales se almacenaron en formol al 5\% con el fin de hacer el análisis y determinar la carga parasitaria. El material fue procesado en el Laboratorio de Parasitología de la Universidad de Córdoba, en Montería. Se utilizó la técnica de observación directa y flotación para análisis de los endoparásitos. Se utilizaron muestras de sangre entera en todos los osos hormigueros gigantes muestreados para realizar la prueba de "gota gruesa" con el fin de determinar hemoparásitos. 
La obtención de los datos se realizó previa anestesia del paciente, y se administró bajo estricta vigilancia del médico veterinario. La obtención de las muestras se llevó a cabo acorde con las condiciones del sitio y manteniendo las normas de bioseguridad establecidas para tal fin. Se tuvo en cuenta la Resolución No. 008430 de 1993 (4 de octubre de 1993, artículo 87, literales c, g y h) del Ministerio de Salud de Colombia. Se cumplió con los requisitos de la legislación sobre la investigación científica en diversidad biológica, que involucra alguna o todas las actividades de recolección, captura, caza, pesca, manipulación del recurso biológico y su manipulación en el territorio nacional. Se obtuvo el permiso de la Corporación Autónoma de la OrinoquíaCorporinoquia, en el departamento del Casanare, de donde se tomaron las muestras correspondientes, de conformidad con el decreto 309, artículo 2A de 2000 del Ministerio de Medio Ambiente y Desarrollo Sostenible de Colombia. Los investigadores de este estudio conocen los "principios éticos de la experimentación animal" enunciados por el International Council for Laboratory Animal Science (ICLAS).

\section{Resultados}

Parásitos gastrointestinales: En total se realizaron 11 exámenes coprológicos en hormigueros gigantes (MT) y 2 en osos meleros (TT), de los cuales siete hormigueros gigantes presentaron carga parasitaria $(63,6 \%)$ y ambos meleros se encontraron positivos (100\%). Los resultados se encuentran en la Tabla 2 . Se identificaron 11 géneros de parásitos gastrointestinales (Fig. 1, 2).

Tabla 2. Parásitos gastrointestinales encontrados en hormigueros gigantes y osos meleros de vida libre.

\begin{tabular}{cl}
\hline \# Individuo & \multicolumn{1}{c}{ Especie de parásito } \\
\hline MT004 & Blastocistis hominis, Coccidia sp. \\
MT005 & Blastocistis hominis \\
MT006 & Quiste de Entoamoeba dispar, quiste de Giardia lamblia \\
MT007 & Huevo de Taenia sp. \\
MT008 & Coccidia sp. \\
MT009 & Isospora sp., larva de Strongyloides sp. \\
MT010 & Huevo de Ascaris lumbricoides, trofozoitos de Chilomastix mesnili \\
TT001 & Trichostrongylus sp., Coccidia sp., Taenia sp. \\
TT002 & Coccidia sp. \\
\hline
\end{tabular}

Los parásitos con mayor prevalencia en osos hormigueros gigantes fueron Coccidia sp (28\%) y Blastocistis hominis (28\%). Los otros parásitos identificados presentaron prevalencias menores: E. dispar $(14,28 \%)$, G. lamblia $(14,28 \%)$, Isospora sp. $(14,28 \%)$, Strongyloides sp. $(14,28 \%)$, Ascaris lumbricoides (14,28\%), Chilomastix mesnili $(14,28 \%)$ $(14,28 \%)$. En cuanto a los osos meleros, la Coccidia fue el parásito más prevalente $(100 \%)$, mientras que Trichostrongylus sp., y Taenia sp., presentaron una prevalencia del $50 \%$.

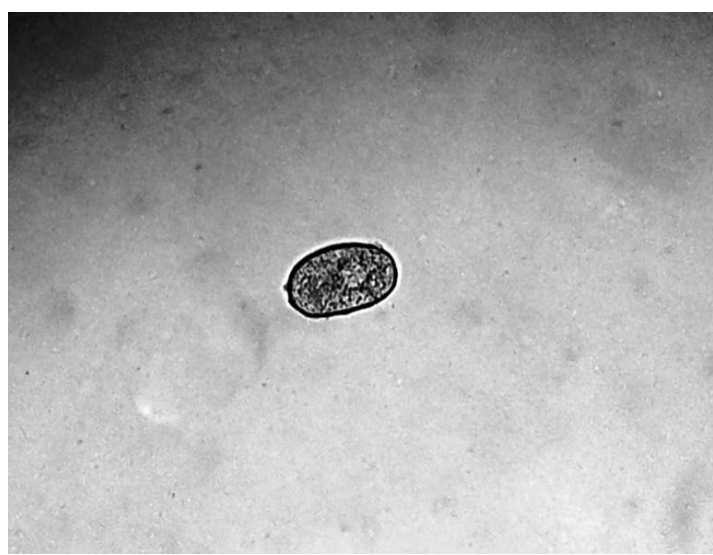

Figura 1. Larva de Strongyloides sp., en oso hormiguero gigante de vida libre.

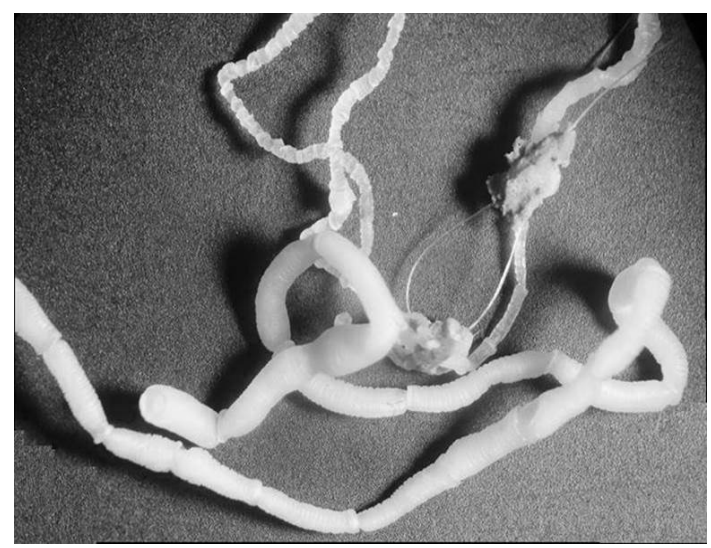

Figura 2. Taenia sp., en oso melero de vida libre

Hemoparásitos: El $27 \%$ de las muestras analizadas $(3 / 11)$ de osos hormigueros gigantes se encontraron positivas a hemoparásitos. Todos los animales positivos presentaron parásitos del género Babesia (Fig. 3).

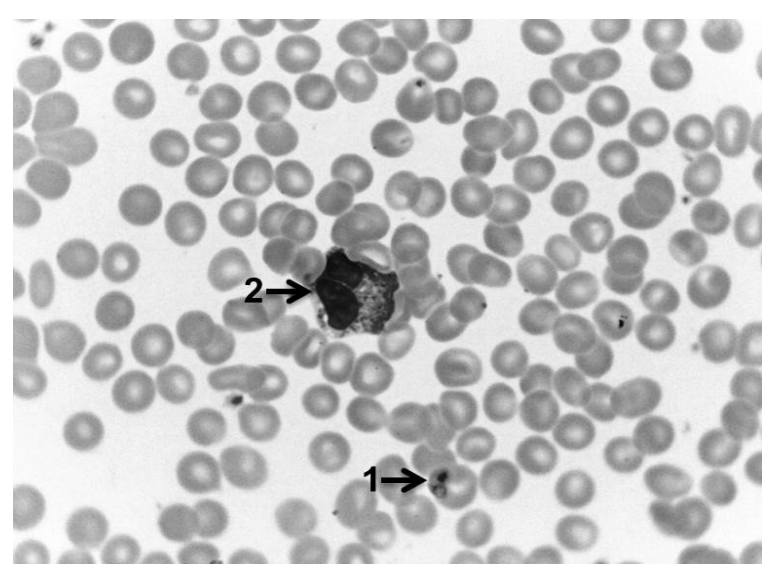

Figura 3. Microfotografía de células hemáticas en hormigueros gigantes. 1. Babesia sp. 2. Polimorfonuclear. Objetivo: $40 x$. 


\section{Discusión}

Este es el primer reporte para algunos parásitos en osos hormigueros gigantes y meleros de vida libre en Colombia. Las formas parásitas monoxenas (Giardia lamblia, Trichostrongylus sp., Entomoeba dispar, Blastocistis hominis, Coccidia sp., Isospora sp., Strongyloides sp., Ascaris lumbricoides, Chilomastix mesnili) fueron predominantes, aunque también se hallaron parásitos de ciclo heteroxeno (Taenia sp.) que necesitan de un hospedador intermediario.

Se considera que el contacto directo de los hormigueros con el suelo es uno de los principales factores de riesgo para el desarrollo de enfermedades, en especial de origen parasitario (DINIZ et al., 1995; IBAÑEZESCRIBANO et al., 2013). Por otro lado, la ingesta incidental de material vegetal (EZQUIAGA et al., 2009), o el consumo de agua contaminada con heces de otras especies de mamíferos presentes en la zona (seres humanos, ganado doméstico, venados (Odocoileus virginianus), chigüiros (Hydrochoerus hydrochaeris), entre otros) podría explicar, entonces, la predominancia de formas monoxenas en ambas especies.

Si bien la dieta de los Vermilingua es estrictamente insectívora, al parecer los insectos componentes de la dieta no cumplen un papel importante en la transmisión de endoparásitos, dada la baja diversidad heteroxenos (una sola especie) presentes. Esta diversidad baja se reportó en otros xenartros, como Zaedyus pichiy, no obstante, esta especie de armadillo ha sido clasificada como omnívora, consumiendo material vegetal que pudiera ser fuente de infección (SUPERINA, 2007; EZQUIAGA et al., 2009).

La mayor prevalencia de parásitos en ambos Vermilinguas correspondió a Coccidia sp. Estos parásitos gastrointestinales son comúnmente encontrados en animales silvestres, ya sea en vida libre o en cautiverio. En el caso puntual de los hormigueros, diversos autores han reportado la presencia de Coccidias (Género Eimeria) en Vermilinguas de vida libre (LAINSON y SHAW, 1982; GARDNER et al., 1991), por lo que se considera que es un parásito común en estas especies.

En M. tridactyla se encontró una prevalencia de $28 \%$ de Blastocistis hominis. Este es uno de los parásitos más frecuente en seres humanos, sin embargo, no ha sido reportado anteriormente en osos hormigueros en Colombia y su clasificación taxonómica es discutida aún. Este parásito gastrointestinal se ha reportado animales silvestres, especialmente primates, como monos araña (Alouatta fusca) y marmosetas (Callithrix jaccus y $C$. penicillata) en Brasil (CARVALHO-FILHO et al., 2006), y se ha señalado que su transmisión se da a través del consumo de agua con pobres condiciones higiénico- sanitarias (STENZEL y BOREHAM, 1986), similares a las que suelen consumir los individuos silvestres en la zona de Pore, lo que explicaría su presencia.

De la misma forma, la vía feco-oral, principalmente por el agua contaminada y consumo accidental de material vegetal, podría ser la potencial fuente de infección para los otros parásitos monoxenos encontrados, como E. dispar, G. lamblia, Isospora sp., Strongyloides sp., Ascaris lumbricoides, Chilomastix mesnili y Trichostrongylus sp. (SAREDI, 2002). De estos, Strongyloides sp., ha sido reportado en M. tridactyla mantenido en cautiverio en Colombia (COPETE et al., 2013) y Brasil (MARINHO y VALDES, 2012), mientras que Entomoeba sp., ha sido reportada en esta misma especie en Brasil (MARINHO y VALDES, 2012), lo que indica que pueden parasitar esta especie ya sea de forma natural o accidental.

De todos los parásitos reportados, solo Taenia sp., presenta un ciclo de vida que implica consistentemente dos huéspedes mamíferos, un huésped definitivo carnívoros u omnívoros (por ejemplo, los cánidos, félidos, viverridos, mustélidos, hyaenids y humanos) y una gran cantidad herbívoros intermediarios (principalmente Artiodáctilos, roedores y lagomorfos) (LOOS-FRANK, 2000). No obstante, es probable que la presencia de este parásito en $T$. tetradactyla y $M$. tridactyla esté causada por otras vías de transmisión.

Se ha reportado que las hormigas pueden participar en los ciclos de transmisión de cisticercoides de Cestodos (HEINZE et al., 1998), y que incluso estos parásitos pueden modificar el comportamiento y la pigmentación de las hormigas para así aumentar su consumo y mejorar la transmisión a sus huéspedes finales (GABRION et al., 1976; SAWADA y HARADA, 1995). Si bien no se cuenta con información al respecto en Colombia, puede que esta sea la causa de la presencia de Taenia sp., en T. tetradactyla dados sus hábitos insectívoros.

En referencia a la presencia de hemoparásitos, al parecer no existen registros previos de Babesia sp, para alguna especie de hormiguero en el país, y los reportes en otros Vermilinguas han ocurrido en Brasil, donde se encontraron 19 individuos positivos a tripanosomatídeos (44\%) y 17 a filarideos (40\%) (MIRANDA, 2012). En la transmisión de Babesia las picaduras de garrapatas juegan un papel importante, y es probable que este parásito sea de ocurrencia frecuente en individuos que habiten en zonas ganaderas, debido a que algunos autores han reportado la presencia común de garrapatas en hormigueros silvestres (MIRANDA, 2012). 
Los avances en las investigaciones parasitológicas de animales silvestres y en particular de xenartros permitirán ampliar el conocimiento taxonómico de su fauna parasitaria, su distribución geográfica y el espectro de especies hospedadoras con el fin último de referenciar áreas, hospedadores y especies parasitarias asociadas (EZQUIAGA et al., 2009). Aun cuando no se cuenta con evidencia suficiente para afirmar que estas infestaciones parasitarias puedan estar afectando de manera severa a los individuos muestreados o que simplemente hagan parte de la flora normal de éstos, los resultados podrán servir de base para próximos estudios a larga escala donde se identifique la carga parasitaria normal de estas especies, información que será útil en procesos de reintroducción, liberación y manejo de animales a nivel nacional.

\section{Agradecimientos.}

Este estudio fue financiado por la Compañía Geopark S.A. dentro del marco del proyecto "Investigación sobre la especie de oso palmero para implementar medidas de conservación en Pore, Casanare". Se agradece a Yonairo Herrera y al Laboratorio de Parasitología de la Universidad de Córdoba, al igual que a Lina Arias, María López y Ernesto Roa por su colaboración.

\section{Referencias}

CARVALHO-FILHO, P.; CARDOZO, S.; RIBEIRO, C.; MEDEIROS, S.; LOPES, C. 2006. Intestinal Protozoa in apprehended New World nonhuman primates. Brazilian Journal of Animal Sciences 43 (3):354-361.

COPETE, M.; RAMÍREZ, G.; OSORIO, H. 2013. Principales helmistos encontrados en un centro de fauna cautiva en Colombia. Boletín Científico Museo de Historia Natural 17 (1):251-257.

DEEM, S. L.; KARESH, W. B.; WEISMAN, W. 2001. Putting theory into practice: wildlife health in conservation. Conservation Biology 15:1224-1233.

DINIZ, L; COSTA, E; OLIVEIRA, P. 1995. Clinical disorders observed in anteaters in captivity. Veterinary research communications 19 (1):409-415.

EZQUIAGA, M. C.; SUPERINA, M.; NAVONE, G.T. 2009. Parásitos intestinales de Zaedyus Pichiy (Xenarthra: Dasypodidae) de Mendoza, Argentina. Mastozoología Neotropical 16(2):309-319.

GARDNER, S.; UPTON, S.; LAMBERT, C.; JORDÁN, O. 1991. Redescription of Eimeria escomeli (Rasteg aieff,1930) from Myrmecophaga tridactyla, and a First Report from Bolivia. Journal of Helminthology 58 (1):16-18.

GABRION, C.; PLATEAUX, L; QUENTIN, C. 1976. Anom otaenia brevis (Clerc, 1902) Fuhrmann, 1908 Cestode Cyclophyllide, parasite de Leptothorax nylanderi (Forster) Hyménoptère, Formicidé. Ann. Parasitology 51:407-420.

HEINZE J.; RÜPPELL O.; FOITZIK S.; BUSCHINGER A. 1998. First record of ants with Cestodes from western North-America. Florida Entomologist 81:122-125.

IBAÑEZ-ESCRIBANO, A.; NOGAL-RUIZ, J.J.; DELCLAUX, M.; MARTINEZ-NEVADO, E.; PONCE-GORDO, F. 2013. Morphological and molecular identification of Tetratrichomonas flagellates from the giant anteater (Myrmecophaga tridactyla). Research in Veterinary Science 95:176-181.

LAINSON, R; SHAW, J. 1982. Coccidia of Brazilian edentates: Eimeriu cyclopei n.sp. from the silky anteater, Cyclopes didactylus and Eimeriu choloepi n.sp. from the two-toed sloth, Choloepus didactylus (Lb.). Systematic Parasitology 4:269-278.

LOOS-FRANK, B. 2000. An update of Verster's (1969) 'Taxonomic revision of the genus Taenia Linnaeus' (Cestoda) in a table format. Systematic Parasitology 45:155-183.

MARINHO A; VALDES S. 2012. Diagnóstico de parásitos grastrointestinales de osos hormigueros gigantes (Myrmecophaga tridactyla, Linnaeus, 1758) en cautiverio y su terapéutica farmacológica reporte de un caso. Clínica Veterinária, Sao Paulo 96:96-97. 
MIRANDA, F. 2012. Manutencáo de tamanduas em cativeiro. Editora Cubo. São Carlos- SP, Brasil.

MIRANDA, F.; DE PAULA, C. 2012. Principais enfermidades em tamanduás cautivos. Págs. 240-255 en: Miranda, F (ed.). Manutencáo de tamanduas em cativeiro. Editora Cubo. São Carlos- SP, Brasil.

RODRÍGUEZ, J.; ALBERICO, M; TRUJILLO, F; JORGENSON J. 2006. Libro Rojo de los Mamíferos de Colombia. Serie Libros Rojos de especies amenazadas de Colombia. Conservación Internacional Colombia and Ministerio de Ambiente, Vivienda y Desarrollo, Bogotá.

SAREDI, N. 2002. Manual práctico de parasitología médica. Laboratorios Andrómaco. Buenos Aires, Argentina.

SAWADA, I.; HARADA, M. 1995. Cestode parasites from some Nepalese mountain shrews. Japanese Journal of Parasitology 44:196-209.

STENZEL D.; BOREHAM. P. 1986. Blastocystis hominis Revisited. Clinical Microbiology Reviews 9:563-84.

SUPERINA, M. 2007. Natural history of the pichi (Zaedyus pichiy) in Mendoza Province, Argentina. Tesis de doctorado. University of New Orleans. USA.

SUPERINA, M; MIRANDA, F; ABBA. A. M. 2010. The 2010 anteater Red List assessment. Edentata 11:96-114.

WOODROFFE R. 1999. Managing disease threats to wild mammals. Animal Conservation 2 (3):85-193. 